\title{
SOBRE O DIMENSIONAMENTO DE PILARES DE AÇO EM SITUAÇÃO DE INCÊNDIO. REVISÃO DA NBR 14323
}

\author{
Valdir Pignatta e Silva ${ }^{(1)}$, Jorge Saúl Suaznábar Velarde ${ }^{(2)}$
}

Resumo

O dimensionamento de pilares de aço à temperatura ambiente conforme a NBR 8800 de 1986 tinha por base as conhecidas múltiplas curvas europeias, depois incorporadas ao Eurocode 3. A ABNT NBR 14323:1999, sobre dimensionamento em incêndio, também seguiu a norma europeia. Havia, pois, similaridade entre as formulações das duas normas. A base escolhida para a nova ABNT NBR 8800:2008, no entanto, foi o AISC, norte-americano. O objetivo deste trabalho é propor que a revisão da NBR 14323 mantenha a base europeia para o dimensionamento de pilares em incêndio. Para conduzir a essa proposição, apresenta-se uma detalhada e didática introdução sobre o comportamento de pilares à temperatura ambiente. São abordados tópicos relacionados à teoria clássica de flambagem, que é válida somente para pilares ideais, e o efeito de imperfeições geométricas e do material. Apresenta-se, também, um estudo simplificado da estabilidade do equilíbrio de pilares com rigidez concentrada. As recomendações normativas são comentadas e é feita uma proposta para a revisão da norma brasileira ABNT NBR 14323:1999. Incluem-se gráficos que permitem, de forma expedita, determinar-se a temperatura crítica de pilares em incêndio.

Palavras-chave: incêndio, pilares, instabilidade.

\section{TEORIA CLÁSSICA DE FLAMBAGEM}

A teoria clássica de flambagem é aplicada somente a pilares ideais, ou seja, que possuem as seguintes características: material com comportamento elástico-linear, homogêneo (sem tensões residuais), barra com extremidades articuladas, sem imperfeições geométricas, força centrada e não ocorre instabilidade local ou por torção. Empregando-se a equação diferencial aproximada da linha elástica, eq. 1 e considerando-se que $\mathrm{M}=\mathrm{N}$. y (figura 1), é possível encontrar a força crítica de flambagem elástica, indicada na eq. 2 , em que $\lambda=\ell / \mathrm{r}$ é o índice de esbeltez, onde $\mathrm{r}=\sqrt{\mathrm{I} / \mathrm{A}}$ é o raio de giração.

$$
\frac{\mathrm{d}^{2} \mathrm{y}}{\mathrm{dx}^{2}}=\frac{-\mathrm{M}}{\mathrm{EI}}
$$

\footnotetext{
${ }^{1}$ Professor Doutor da Escola Politécnica da Universidade de São Paulo. Escola Politécnica da Universidade de São Paulo, Departamento de Engenharia de Estruturas e Geotécnica - Av. Prof. Almeida Prado, trav2, n271 Edifício da Engenharia Civil - Cidade Universitária - 05508-900, São Paulo, Brasil email: valpigss@ usp.br

${ }^{2}$ Mestre em Engenharia Civil pela Escola Politécnica da Universidade de São Paulo. Email: jorgesauls@hotmail.com
} 


$$
\mathrm{N}_{\mathrm{cr}}=\frac{\pi^{2} \mathrm{EI}}{\ell^{2}}=\frac{\pi^{2} \mathrm{EA}}{\lambda^{2}}
$$

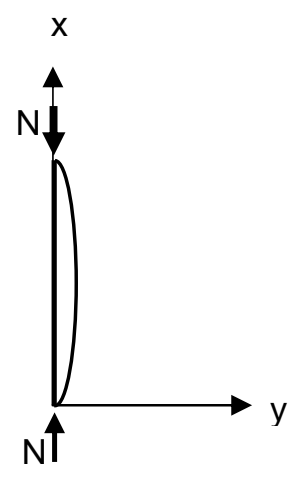

Figura 1: Barra sob compressão

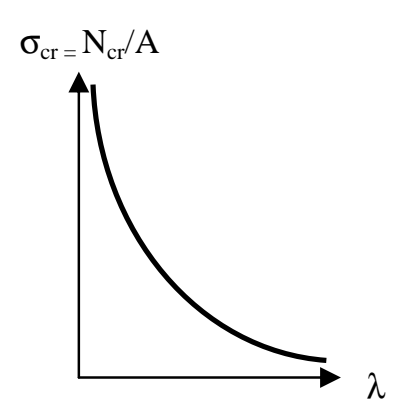

Figura 2: Hipérbole de Euler $\left(\sigma_{\mathrm{cr}} \times \lambda\right)$

Na figura 2, é mostrado o gráfico $\sigma_{\mathrm{cr}} \times \lambda$, conhecido como hipérbole de Euler, em que $\sigma_{\mathrm{cr}}=$ $\mathrm{N}_{\mathrm{cr}} / \mathrm{A}$.

A eq. 2 foi deduzida a partir da expressão aproximado da curvatura, eq. 1. Utilizando-se a equação simplificada eq 1 , somente é possível deduzir a força que provoca flambagem, mas não a configuração pós-crítica, ou seja, o valor de y fica indeterminado. Empregando uma expressão mais precisa para a curvatura, eq. 3, é possível encontrar a relação entre a força normal aplicada e o deslocamento no centro da peça conforme figura 3, para material elástico-linear.

$$
\frac{1}{\rho}=\frac{M}{E I}=\frac{-y^{\prime \prime}}{\left(1+y^{\prime 2}\right)^{3 / 2}}
$$

Como se pode observar na figura 3, após $\mathrm{N}_{\mathrm{cr}}$ há duas soluções possíveis de equilíbrio. Uma, instável, em que a barra permanece na posição indeformada lateralmente e outro, estável, em que a barra se deforma a procura da estabilidade, porém com deslocamentos incompatíveis com o uso normal para uma construção civil. À ocorrência do ponto de bifurcação $\left(\mathrm{N}_{\mathrm{cr}}\right)$ na trajetória de equilíbrio apresentada na figura 3 denomina-se "flambagem". O máximo valor de deslocamento lateral, $\mathrm{y}_{\text {máx }}$, para valores de $\mathrm{N}$ pouco superiores a $\mathrm{N}_{\mathrm{cr}}$ é visualizado na figura 4 (Fruchtengarten 1988). Para $\mathrm{N}>\mathrm{N}_{\mathrm{cr}}$, y é muito elevado para a construção civil, conduzindo as peças usuais à sua capacidade resistente. A flambagem corresponde, portanto, a um Estado Limite Último, ou seja, $\mathrm{N}_{\mathrm{R}}=\mathrm{N}_{\mathrm{cr}}$ em que $\mathrm{N}_{\mathrm{R}}$ é a força normal resistente. 


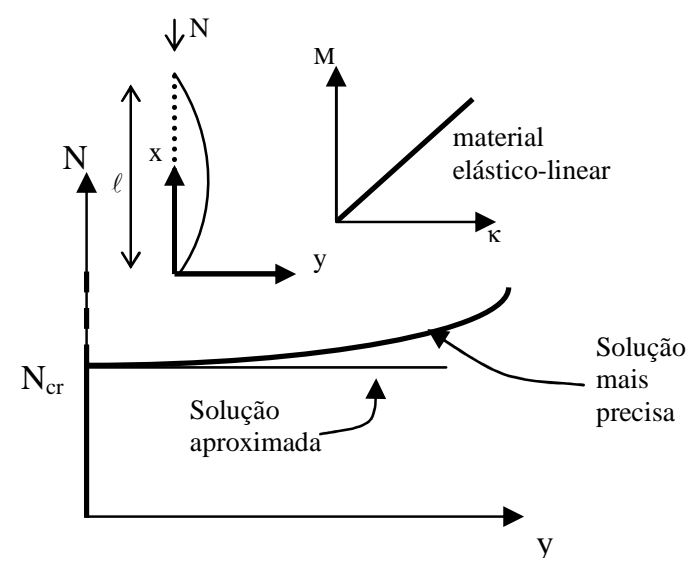

Figura 3: Gráfico N x y para material elástico-linear

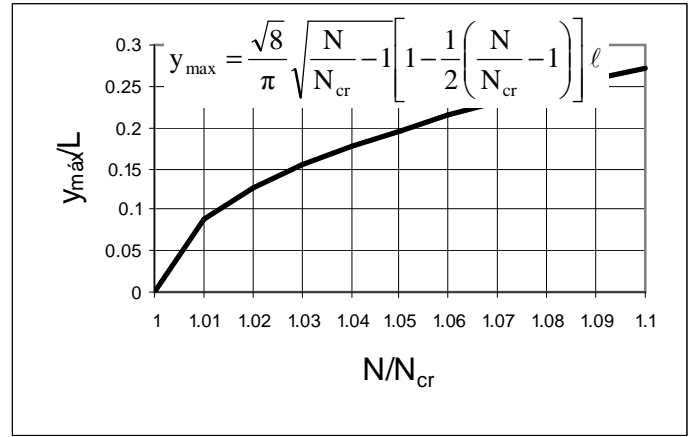

Figura 4: Relação entre força aplicada e deslocamento máximo lateral

Para outras condições de vínculo, segundo a teoria clássica da estabilidade elástica, a força crítica pode ser calculada por meio da eq. 2 substituindo-se $\ell$ por $\mathrm{k} \ell$, onde $\mathrm{k}$ o parâmetro de flambagem que depende das condições de vínculo. No entanto, em casos práticos, as barras sempre estarão submetidas à flexocompressão, pois, a compressão centrada é sempre acompanhada de imperfeições geométricas. Nesse caso, a ABNT NBR 8800:2008 recomenda que se adote $\mathrm{k}=1$. A antiga estratégia de usar valores de $\mathrm{k}$ superiores a 1,0 foi substituída pela consideração de imperfeições iniciais equivalentes, geométricas e do material.

\section{MATERIAL DE COMPORTAMENTO ELASTOFRÁGIL}

\subsection{Ausência de imperfeição geométrica}

O diagrama tensão-deformação de um material elastofrágil é apresentado na figura 5. A esbeltez limite entre regime elástico e ruptura, $\lambda_{\text {pi }}$, pode ser determinada igualando-se a força que provoca flambagem, $\mathrm{N}_{\mathrm{cr}}=\pi^{2} \mathrm{EA} / \lambda_{\mathrm{pi}}^{2}$, àquela que provoca a ruptura, $\mathrm{N}_{\mathrm{y}}=\mathrm{f}_{\mathrm{y}} \mathrm{A}$, resultando a eq. 4.

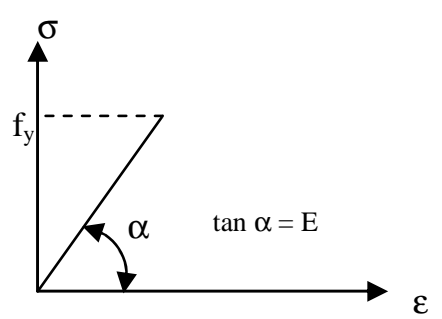

Figura 5: Diagrama tensão-deformação de um Figura 6: Gráfico $\chi \times \lambda_{0}$ para material elastofrágil, material elastofrágil

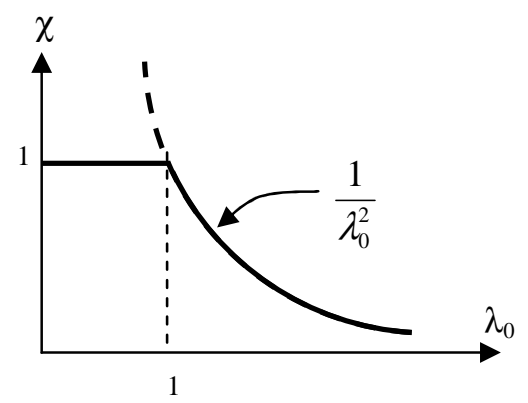

sem imperfeição geométrica 


$$
\lambda_{\mathrm{pi}}=\sqrt{\frac{\pi^{2} \mathrm{E}}{\mathrm{f}_{\mathrm{y}}}}
$$

Considerando-se a eq. 5 na eq. 4 , tem-se a eq. 6 , na qual $\mathrm{N}_{\mathrm{R}}$ é a força normal resistente de pilar.

$$
\begin{aligned}
& \lambda_{0}=\frac{\lambda}{\lambda_{\mathrm{pi}}}=\frac{\lambda}{\sqrt{\frac{\pi^{2} \mathrm{E}}{\mathrm{f}_{\mathrm{y}}}}} \\
& \lambda \leq \lambda_{\mathrm{pi}} \rightarrow \lambda_{0} \leq 1 \quad \mathrm{~N}_{\mathrm{R}}=\mathrm{N}_{\mathrm{y}}=\mathrm{f}_{\mathrm{y}} \mathrm{A} \therefore \frac{\mathrm{N}_{\mathrm{R}}}{\mathrm{N}_{\mathrm{y}}}=1 \\
& \lambda>\lambda_{\mathrm{pi}} \rightarrow \lambda_{0}>1 \quad \mathrm{~N}_{\mathrm{R}}=\mathrm{N}_{\mathrm{cr}}=\frac{\pi^{2} \mathrm{EI}}{\ell^{2}} \therefore \frac{\mathrm{N}_{\mathrm{R}}}{\mathrm{N}_{\mathrm{y}}}=\frac{\pi^{2} \mathrm{E}}{\lambda^{2} \mathrm{f}_{\mathrm{y}}}=\frac{1}{\lambda_{0}^{2}}
\end{aligned}
$$

Denominando-se $\chi=\mathrm{N}_{\mathrm{R}} / \mathrm{N}_{\mathrm{y}}$, pode-se ver na figura 6 a relação $\chi \times \lambda_{0}$.

\subsection{Efeito da imperfeição geométrica}

Como será visto no item 4, na presença de imperfeição não ocorre o fenômeno da flambagem. Trata-se de um problema de flexão composta, no qual se deve considerar a amplificação de flecha (eq. 9) e, portanto, de momento fletor na barra, devido à não linearidade geométrica. No entanto, é possível se reduzir o dimensionamento à flexão composta em uma compressão simples, por meio de um fator de redução da capacidade resistente, $\chi$. O deslocamento total (ver figura 18) é dado pela eq. 7 em que $\left(1-\mathrm{N} / \mathrm{N}_{\mathrm{cr}}\right)^{-1}$ é o fator de amplificação de flechas.

$$
\delta_{\mathrm{t}}=\delta_{0}+\delta=\frac{\delta_{0}}{\left(1-\frac{\mathrm{N}}{\mathrm{N}_{\mathrm{cr}}}\right)}
$$

Em regime elástico a tensão máxima atuante na seção transversal é determinada pela eq. 8.

$$
\sigma_{\max }=\frac{\mathrm{N}}{\mathrm{A}}+\frac{\mathrm{M}}{\mathrm{W}}
$$

eq. 8 


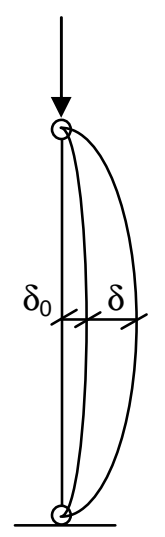

Figura 7: Pilar com imperfeição inicial

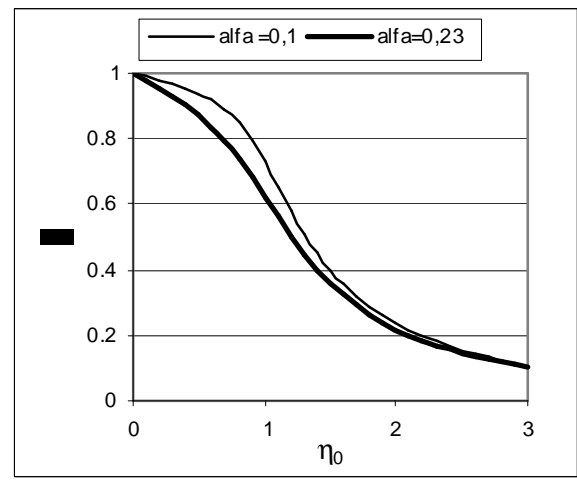

Figura 8: Relação entre $\chi$ e $\lambda_{0}$ para material elastofrágil com imperfeição geométrica em função de $\alpha$

Substituindo-se M por $\mathrm{N} \delta_{\mathrm{t}}$ e considerando-se a eq. 7, tem-se a eq. 9

$$
\sigma_{\max }=\frac{\mathrm{N}}{\mathrm{A}}+\frac{\mathrm{N}}{\mathrm{W}} \frac{\delta_{0}}{\left(1-\frac{\mathrm{N}}{\mathrm{N}_{\mathrm{cr}}}\right)}
$$

Denominando-se $\mathrm{N} /\left(\mathrm{Af}_{\mathrm{y}}\right)=\chi$ e considerando-se $\sigma_{\text {máx }}=\mathrm{f}_{\mathrm{y}}$, tem-se a eq. 10 .

$$
\mathrm{f}_{\mathrm{y}}=\chi \mathrm{f}_{\mathrm{y}}+\frac{\chi \mathrm{Af}_{\mathrm{y}}}{\mathrm{W}} \frac{\delta_{0}}{\left(1-\frac{\mathrm{N}}{\mathrm{N}_{\mathrm{cr}}}\right)}
$$

Lembrando da eq. 2 e de que $\mathrm{N}=\chi \mathrm{A} \mathrm{f}_{\mathrm{y}}$, tem-se a eq. 11

$$
\frac{\mathrm{N}}{\mathrm{N}_{\mathrm{cr}}}=\chi \lambda_{0}^{2}
$$

Substituindo-se a eq. 11 na eq. 10, obtém-se a eq. 12 .

$$
\chi^{2} \lambda_{0}^{2}-\chi\left(1+\lambda_{0}^{2}+\frac{\mathrm{A} \delta_{0}}{\mathrm{~W}}\right)+1=0
$$

Resolvendo a eq. 12, tem-se a eq. 13, conhecida como expressão de Ayrton-Perry (1886).

$$
\chi=\frac{\left(1+\frac{\delta_{0} \mathrm{~A}}{\mathrm{~W}}+\lambda_{0}^{2}\right)-\sqrt{\left(1+\frac{\delta_{0} \mathrm{~A}}{\mathrm{~W}}+\lambda_{0}^{2}\right)^{2}-4 \lambda_{0}^{2}}}{2 \lambda_{0}^{2}}
$$


Escrevendo-se a curvatura inicial (flecha) como função do comprimento da peça, $\delta_{0}=\ell / \mathrm{n}$ e lembrando-se da eq. 5 em que se substitui $\lambda$ por $\ell / r$, tem-se a eq. 14 .

$$
\frac{\delta_{0} \mathrm{~A}}{\mathrm{~W}}=\frac{\lambda_{0}}{\mathrm{n}} \frac{\mathrm{Ar}}{\mathrm{W}} \sqrt{\frac{\pi^{2} \mathrm{E}}{\mathrm{f}_{\mathrm{y}}}}
$$

Aplicando-se a eq. 14 na eq. 13 obtém-se a eq. 15, conhecida como expressão de PerryRobertson, onde $\alpha$ (eq. 16) é um fator de imperfeição.

$$
\begin{gathered}
\chi=\frac{\left(1+\alpha \lambda_{0}+\lambda_{0}^{2}\right)-\sqrt{\left(1+\alpha \lambda_{0}+\lambda_{0}^{2}\right)^{2}-4 \lambda_{0}^{2}}}{2 \lambda_{0}^{2}} \\
\alpha=\frac{\mathrm{Ar}}{\mathrm{nW}} \sqrt{\frac{\pi^{2} \mathrm{E}}{\mathrm{f}_{\mathrm{y}}}}
\end{gathered}
$$

Nota: Segundo a ABNT NBR 5884:2005 a tolerância de fabricação para flecha longitudinal é L/1000, enquanto a curva de dimensionamento adotada pela ABNT NBR 8800:2008 considera L/1500 de imperfeição inicial (PIMENTA (1997))

Adotando-se: $1000 \leq \mathrm{n} \leq 1500 ; 80 \leq \sqrt{\pi^{2} \mathrm{E} / \mathrm{f}_{\mathrm{y}}} \leq 90$ e sabendo-se que para a maioria dos perfis laminados comercializados no Brasil $1,95 \leq \mathrm{Ar}_{\mathrm{y}} / \mathrm{W}_{\mathrm{y}} \leq 2,5$, resulta: $0,1 \leq \alpha \leq 0,23$.

Nota: em 1925, Robertson (apud Reis e Camotim 2001) admitiu: $\delta_{0} \mathrm{~A} / \mathrm{W}=0,003 \lambda$ o que corresponde a aproximadamente $\alpha \sim 0,25$. Na figura 8 é apresentada a relação entre $\chi$ e $\lambda_{0} \mathrm{em}$ função de $\alpha$.

\section{MATERIAL COM COMPORTAMENTO NÃO LINEAR}

\subsection{Material elastoplástico perfeito (sem imperfeição geométrica)}

O diagrama tensão-deformação de um material elastoplástico perfeito é apresentado na figura 9. Da mesma forma que para o material elastofrágil, encontra-se a relação apresentada à figura 10, onde $\mathrm{N}_{\mathrm{R}}$ é a força normal resistente do pilar constituído de material de comportamento elastoplástico perfeito. 


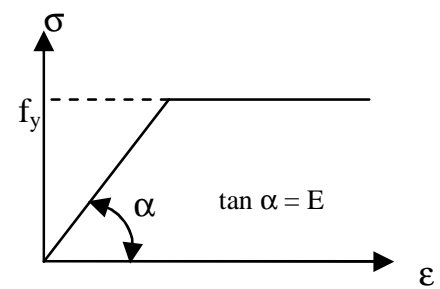

Figura 9: Diagrama tensão-deformação de um material elastoplástico perfeito

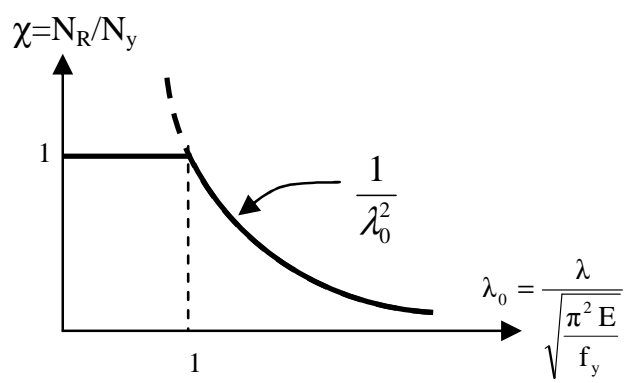

Figura 10: Gráfico $\mathrm{N}_{\mathrm{R}} / \mathrm{N}_{\mathrm{y}} \quad \mathrm{x} \quad \lambda_{0}$ para material elastoplástico perfeito, sem imperfeição geométrica

\subsection{Material elastoplástico não linear (sem imperfeição geométrica)}

Para os materiais com comportamento elastoplástico não linear que seguem um diagrama $\underline{\sigma}$ x $\varepsilon$, conforme figura 11, deve-se criar uma esbeltez limite, $\lambda_{\mathrm{r}}$, para aplicação da expressão de Euler, eq. 2. $\lambda_{\mathrm{r}}$ pode ser determinada igualando-se a força que provoca flambagem, $\mathrm{N}_{\mathrm{cr}}=\pi^{2} \mathrm{EA} / \lambda_{\mathrm{r}}^{2}$, àquela que provoca a tensão correspondente ao limite de proporcionalidade $\left(\sigma_{\mathrm{p}} \mathrm{A}\right)$, o que resulta na eq. 17.

$$
\lambda_{\mathrm{r}}=\sqrt{\frac{\pi^{2} \mathrm{E}}{\sigma_{\mathrm{p}}}}
$$

Pela teoria do módulo tangente de Engesser, substitui-se o módulo de elasticidade, E, pelo módulo de elasticidade tangente, $\mathrm{E}_{\mathrm{t}}$, na eq. 2.

Assim, tem-se: $N_{R}=\frac{\pi^{2} E_{t} I}{\ell^{2}}=\frac{\pi^{2} E_{t} I}{\lambda^{2} r^{2}}=\frac{\pi^{2} E_{t} A}{\lambda^{2}}=\frac{E_{t} A_{y}}{\lambda_{0}^{2} E}=\frac{N_{y} E_{t} / E}{\lambda_{0}^{2}}$ e se obtém a eq. 18, em que $\chi=\mathrm{N}_{\mathrm{R}} / \mathrm{N}_{\mathrm{y}}$ e $\lambda_{0}$ conforme eq. 5 .

$$
\chi=\frac{\mathrm{E}_{\mathrm{t}} / \mathrm{E}}{\lambda_{0}^{2}}
$$

O gráfico $\chi$ x $\lambda_{0}$ é ilustrado na figura 12. Lembra-se que: $\frac{\lambda_{\mathrm{r}}}{\sqrt{\pi^{2} E / f_{y}}}=\frac{\sqrt{\pi^{2} E / \sigma_{p}}}{\sqrt{\pi^{2} E / f_{y}}}=\sqrt{\frac{\mathrm{f}_{\mathrm{y}}}{\sigma_{\mathrm{p}}}}$. 

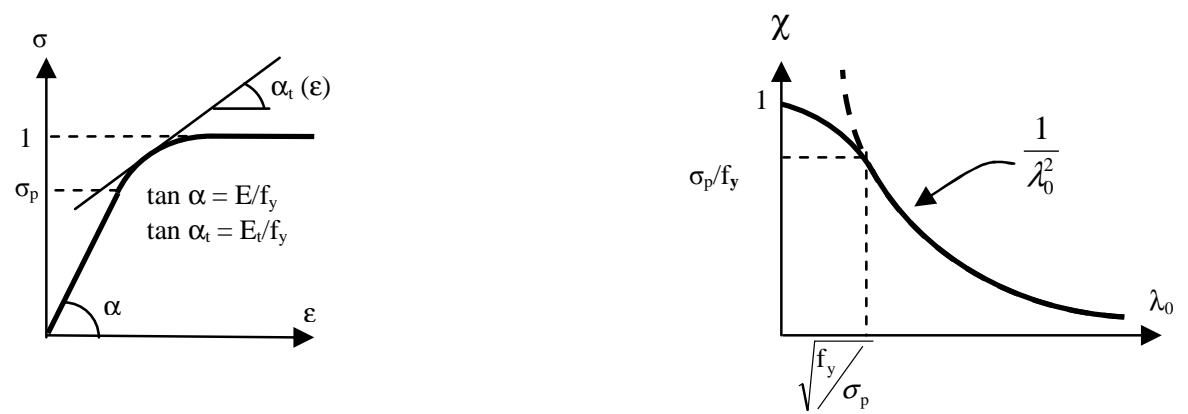

Figura 11: Gráfico $N / N_{y}$ x $\varepsilon$ para material Figura 12: Gráfico $\mathrm{N}_{\mathrm{R}} / \mathrm{N}_{\mathrm{y}} \times \lambda_{0}$ para material com elastoplástico não linear comportamento elastoplástico não linear, sem imperfeição geométrica

\subsection{O efeito das tensões residuais}

O diagrama tensão x deformação de uma peça com tensões residuais é apresentado na figura 13. Nesse caso, $\sigma_{\mathrm{p}}=\mathrm{f}_{\mathrm{y}}-\sigma_{\mathrm{r}}$, sendo $\sigma_{\mathrm{r}}$ a tensão residual. A força crítica pode ser determinada conforme a eq. 19, em que $(\mathrm{EI})_{\mathrm{ef}}=\int_{\mathrm{A}} \mathrm{E}_{\mathrm{t}} \mathrm{x}^{2} \mathrm{dA}$.

$$
\mathrm{N}_{\mathrm{cr}}=\frac{\pi^{2}(\mathrm{EI})_{\mathrm{ef}}}{\ell^{2}}
$$

Para material elastoplástico perfeito, $(\mathrm{EI})_{\mathrm{ef}}=\mathrm{E} \int_{\mathrm{A}} \mathrm{x}^{2} \mathrm{dA}=\mathrm{EI} \mathrm{ef}_{\mathrm{ef}}$, portanto, $\mathrm{N}_{\mathrm{cr}}=\pi^{2} \mathrm{EI}_{\mathrm{ef}} / \ell^{2}$.

Como se pode observar na figura 14, para cada seção transversal deve haver duas curvas $\chi \times \lambda_{0}$, uma para a direção x-x e outra para $y-y$.

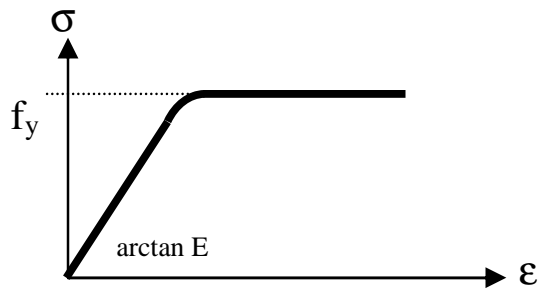

(a)

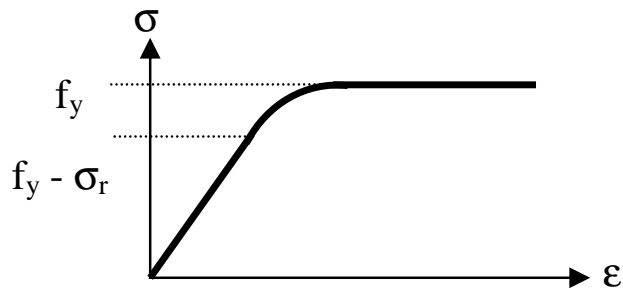

(b)

Figura 13: Diagrama tensão-deformação para (a) aço virgem (b) aço com tensão residual 


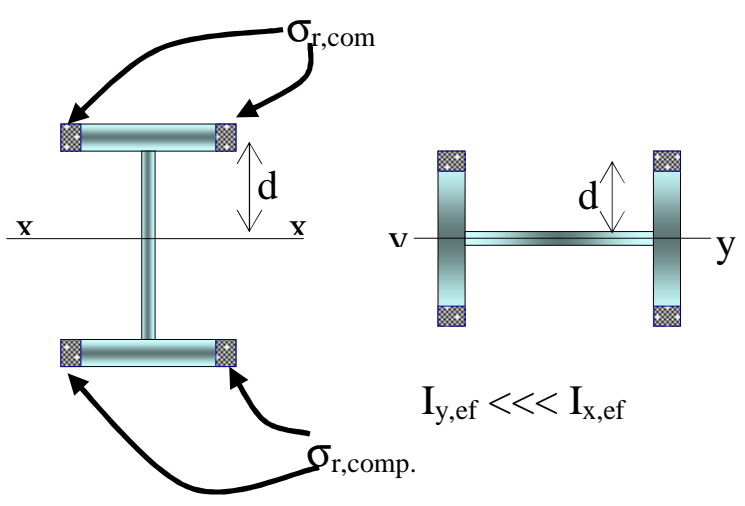

Figura 14: Efeito das tensões residuais

\section{ESTABILIDADE DO EQUILÍBRIO}

Para se analisar o caso geral incluindo não linearidade geométrica (imperfeição) e do material, serão utilizados alguns conceitos básicos da Teoria Geral da Estabilidade.

A energia potencial total de um sistema estrutural (V) pode ser definida como a diferença entre a energia potencial dos esforços internos ou energia de deformação (U) e a energia potencial dos esforços externos (W). Segundo o teorema de Lagrange-Dirichlet: "É condição suficiente para a estabilidade de equilíbrio de uma configuração de um sistema que a energia potencial total seja mínima $(\partial \mathrm{V} / \partial \varphi=0)$. Esse teorema, proposto por Lagrange e demonstrado por Dirichlet, é válido para sistemas conservativos e discretos e será aplicado para casos simples (figura 15) de um pilar com rigidez concentrada na base e imperfeição simulada por meio de um ângulo $\varphi_{0}$. Nas figuras 16 a 19 apresentam-se os diagramas $\mathrm{N}$ x $\varphi$ para os 4 casos analisados conforme Tabela 1.

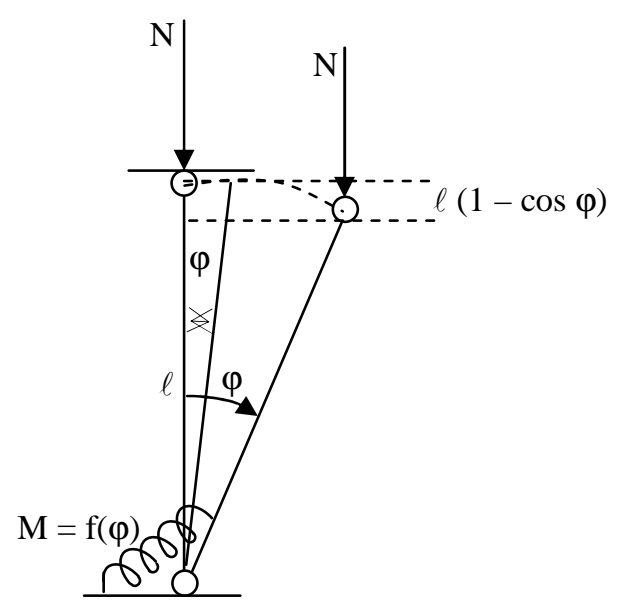

Figura 15: Barra sujeita à força normal 
Tabela 1: Análise da estabilidade do equilíbrio

\begin{tabular}{|c|c|}
\hline $\begin{array}{l}\text { Caso I - Sem imperfeição geométrica e material com } \\
\text { comportamento linear: } \varphi_{0}=0 ; \mathrm{f}(\varphi)=\mathrm{K} \varphi \\
\mathrm{U}=\int_{0}^{\varphi} \mathrm{K} \varphi=\frac{\mathrm{K} \varphi^{2}}{2} \\
\mathrm{~W}=\mathrm{N} \ell(1-\cos \varphi) \\
\mathrm{V}=\mathrm{U}-\mathrm{W}=\frac{\mathrm{K} \varphi^{2}}{2}-\mathrm{N} \ell(1-\cos \varphi) \\
\frac{\partial \mathrm{V}}{\partial \varphi}=\mathrm{K} \varphi-\mathrm{N} \ell \sin \varphi=0 \\
\mathrm{~N}=\frac{\mathrm{K}}{\ell} \frac{\varphi}{\sin \varphi}\end{array}$ & $\begin{array}{l}\text { Caso II - Com imperfeição geométrica e material com } \\
\text { comportamento linear: } \varphi_{0} \neq 0 ; \mathrm{f}(\varphi)=\mathrm{K}\left(\varphi-\varphi_{0}\right) \\
\mathrm{U}=\int_{\varphi_{0}}^{\varphi} \mathrm{K}\left(\varphi-\varphi_{0}\right)=\frac{\mathrm{K}\left(\varphi-\varphi_{0}\right)^{2}}{2} \\
\mathrm{~W}=\mathrm{N} \ell\left[\cos \varphi_{0}-\cos \varphi\right] \\
\mathrm{V}=\mathrm{U}-\mathrm{W}=\frac{\mathrm{K}\left(\varphi-\varphi_{0}\right)^{2}}{2}-\mathrm{N} \ell\left[\cos \varphi_{0}-\cos \varphi\right] \\
\frac{\partial \mathrm{V}}{\partial \varphi}=\mathrm{K}\left(\varphi-\varphi_{0}\right)+\mathrm{N} \ell \sin (\varphi)=0 \\
\mathrm{~N}=\frac{\mathrm{K}}{\ell} \frac{\varphi-\varphi_{0}}{\sin \varphi}\end{array}$ \\
\hline $\begin{array}{l}\text { Caso III - Sem imperfeição geométrica e material cc } \\
\text { comportamento não linear: } \varphi_{0}=0 ; \mathrm{f}(\varphi)=\mathrm{K} \varphi\left(1-\varphi^{2}\right) \\
\mathrm{U}=\int_{0}^{\varphi} \mathrm{K} \varphi\left(1-\varphi^{2}\right)=\mathrm{K}\left(\frac{\varphi^{2}}{2}-\frac{\varphi^{4}}{4}\right) \\
\mathrm{W}=\mathrm{N} \ell(1-\cos \varphi) \\
\mathrm{V}=\mathrm{U}-\mathrm{W}=\mathrm{K}\left(\frac{\varphi^{2}}{2}-\frac{\varphi^{4}}{4}\right)-\mathrm{N} \ell(1-\cos \varphi) \\
\frac{\partial \mathrm{V}}{\partial \varphi}=\mathrm{K}\left(\varphi-\varphi^{3}\right)-\mathrm{N} \ell \sin \varphi=0 \\
\mathrm{~N}=\frac{\mathrm{K}}{\ell} \frac{\varphi-\varphi^{3}}{\sin \varphi}\end{array}$ & $\begin{array}{l}\text { Caso IV - Com imperfeição geométrica e material com } \\
\text { comportamento não linear: } \varphi_{0} \neq 0 ; \mathrm{f}(\varphi)=\mathrm{K}\left(\varphi-\varphi_{0}\right)\left[1-\left(\varphi-\varphi_{0}\right)^{2}\right] \\
\mathrm{U}=\int_{\varphi_{0}}^{\varphi} \mathrm{K}\left(\varphi-\varphi_{0}\right)\left[1-\left(\varphi-\varphi_{0}\right)^{2}\right]=\mathrm{K}\left(\frac{\left(\varphi-\varphi_{0}\right)^{2}}{2}-\frac{\left(\varphi-\varphi_{0}\right)^{4}}{4}\right) \\
\mathrm{W}=\mathrm{N} \ell\left[\cos \varphi_{0}-\cos \varphi\right] \\
\mathrm{V}=\mathrm{U}-\mathrm{W}=\mathrm{K}\left(\frac{\left(\varphi-\varphi_{0}\right)^{2}}{2}-\frac{\left(\varphi-\varphi_{0}\right)^{4}}{4}\right)-N \ell\left(\cos \varphi_{0}-\cos \varphi\right) \\
\frac{\partial \mathrm{V}}{\partial \varphi}=\mathrm{K}\left[\left(\varphi-\varphi_{0}\right)-\left(\varphi-\varphi_{0}\right)^{3}\right]-\mathrm{N} \ell \sin \varphi=0 \\
\mathrm{~N}=\frac{\mathrm{K}}{\ell} \frac{\left[\left(\varphi-\varphi_{0}\right)-\left(\varphi-\varphi_{0}\right)^{3}\right]}{\sin \varphi}\end{array}$ \\
\hline
\end{tabular}

As conclusões observadas nas figuras 16 a 19 podem ser generalizadas para pilares quaisquer. A figura 20 apresenta a relação entre a força normal aplicada e o deslocamento no centro da peça, para materiais de comportamento linear e não linear. Como se pode notar, para pilares com imperfeições, não ocorre bifurcação de equilíbrio no caso de materiais elástico-lineares e, portanto, não ocorre flambagem e sim flexão composta. No caso de materiais de comportamento não linear, também não ocorre ponto de bifurcação, mas há instabilidade a partir do ponto em que não há solução para equilíbrio. Esse ponto é denominado "ponto limite".

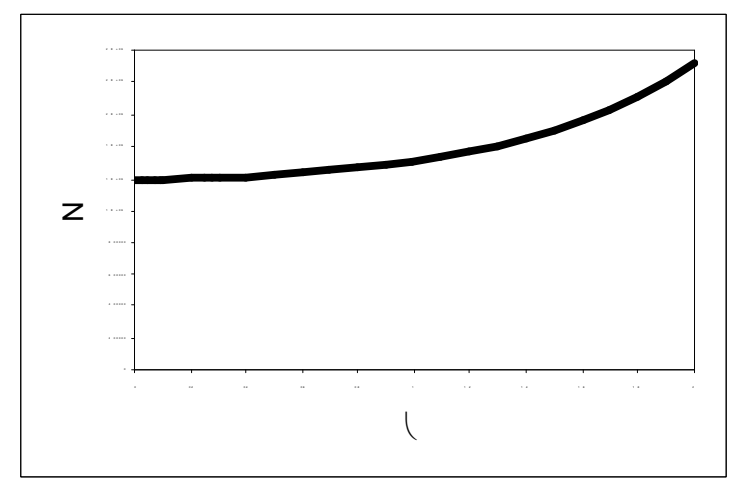

Figura 16: Diagrama N x $\varphi$ para pilar ideal

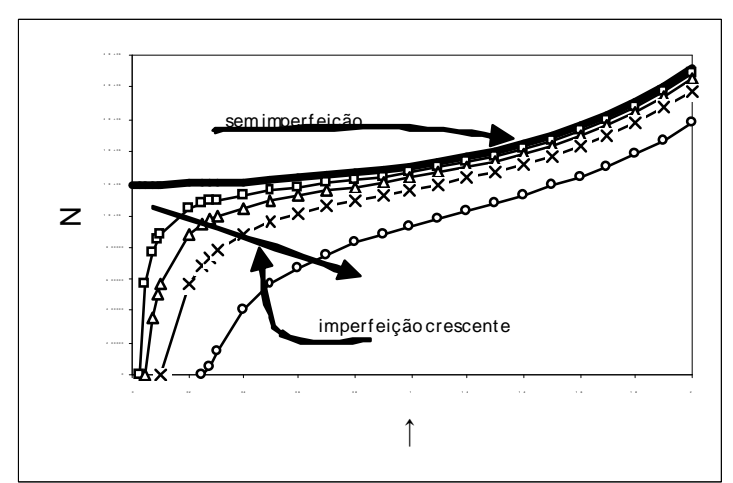

Figura 17: Diagrama $\mathrm{N} \times \varphi$ para pilar com imperfeição geométrica e material de comportamento não linear 


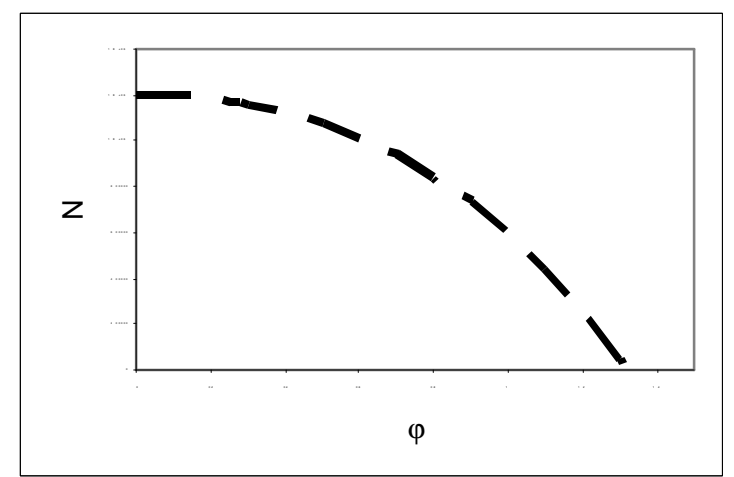

Figura 18: Diagrama $\mathrm{N}$ x $\varphi$ para pilar sem imperfeição com material de comportamento não linear

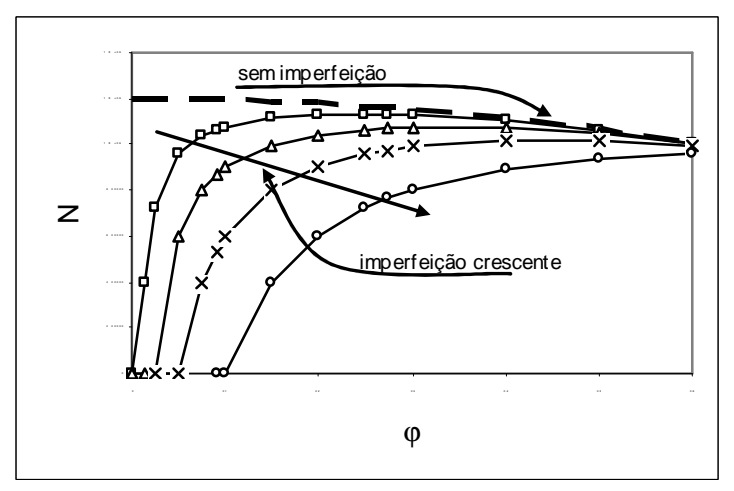

Figura 19: Diagrama N $\mathrm{x} \varphi$ para pilar com imperfeição e material de comportamento não linear
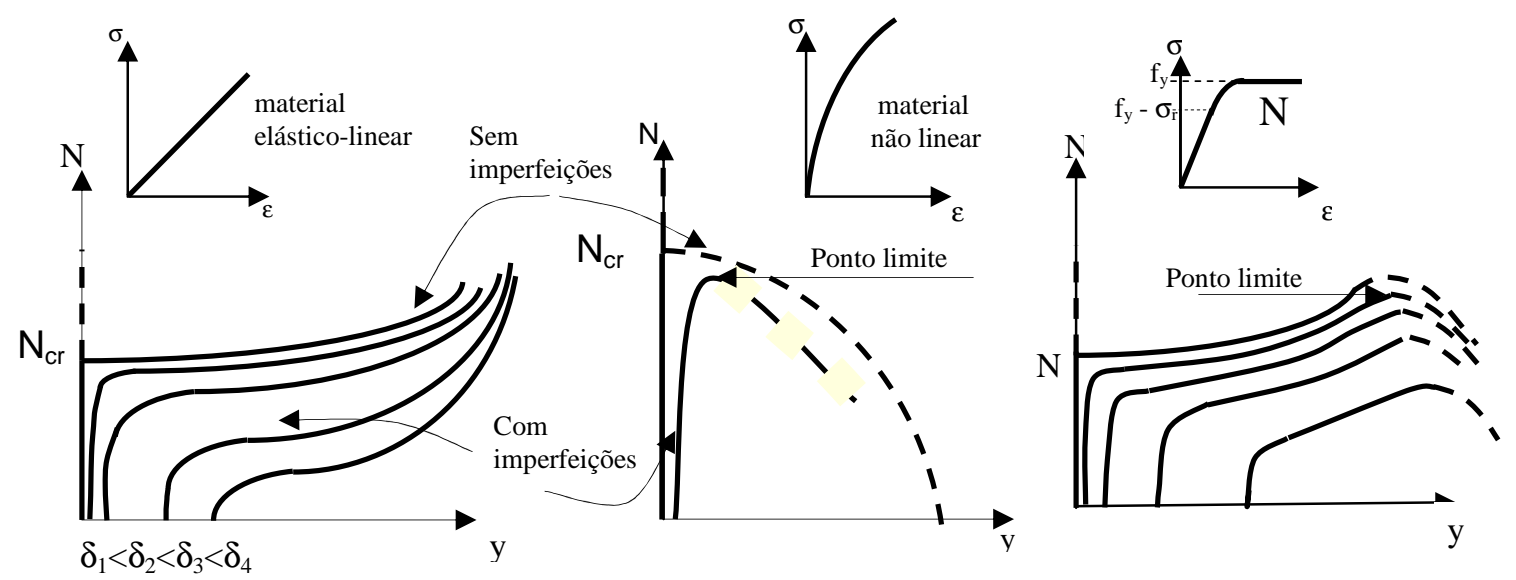

Figura 20: Gráfico N x y de pilares com imperfeições geométricas

\section{CURVAS PARA DIMENSIONAMENTO}

A expressão de Perry-Robertson (eq. 15) corresponde à análise elástica. Com base na expressão de Perry, Rondal; Maquoi (1979) propuseram uma curva incluindo efeitos não elásticos. Ainda em 1979, essa curva foi modificada por J. Rondal e R. Maquoi, apud Janss; Minne (1981), resultando a eq. 20, que foi adotada pela norma europeia Eurocode 3. A eq. 20 é a própria eq. 15 rearranjada, substituindo $\lambda_{0}$ por $\left(\lambda_{0}-0,2\right)$.

$$
\begin{aligned}
& \text { Para } \lambda_{0} \leq 0,2 \quad \chi=1,0 \\
& \text { Para } \lambda_{0}>0,2 \quad \chi=\frac{1}{0,5\left[1+\alpha\left(\lambda_{0}-0,2\right)+\lambda_{0}^{2}\right]+\sqrt{\left[0,5\left(1+\alpha\left(\lambda_{0}-0,2\right)+\lambda_{0}^{2}\right)\right]^{2}-\lambda_{0}^{2}}}
\end{aligned}
$$


Com base em inúmeros ensaios experimentais e numéricos, a eq. 20 foi calibrada a fim de incluir os efeitos das imperfeições geométricas, das tensões residuais e da plasticidade (Reis; Camotin 2001) no valor de $\alpha$, que pode ser entendido como imperfeição inicial "equivalente". Em vista da variabilidade dos resultados, o Eurocode 3 apresenta 4 curvas que se diferenciam em função de $\alpha$, o qual varia entre 0,21 e 0,76 . Na figura 21 apresenta-se a relação entre $\chi$ e $\lambda_{0}$ conforme o Eurocode 3 e conforme a eq. 15, válida para material elástico-linear (item 2.2 deste texto) $\operatorname{com} \alpha=0,15$. Nota-se que as grandes alterações conceituais inseridas pelo Eurocode 3 não afetam demasiadamente a aparência da curva.

A ABNT NBR 8800:2008, inspirada no AISC (2005), recomenda uma curva única para dimensionamento de pilares, conforme eq. 21.

$$
\begin{aligned}
& \text { para } \lambda_{0} \leq 1,5 \rightarrow \chi=0,658^{\lambda_{0}^{2}} \\
& \text { para } \lambda_{0}>1,5 \rightarrow \chi=\frac{0,877}{\lambda_{0}^{2}}
\end{aligned}
$$

Na figura 22 apresentam-se as curvas $\chi$ e $\lambda_{0}$ conforme NBR 8800 e Eurocode 3 (2003). Como se nota, as diferenças são acentuadas entre as normas. No entanto, se observarmos a figura 23, essas diferenças são reduzidas na prática de projeto, em vista de que o coeficiente de ponderação das resistências $\gamma$ vale 1,1 na NBR 8800:2008 e 1,0 no Eurocode 3.

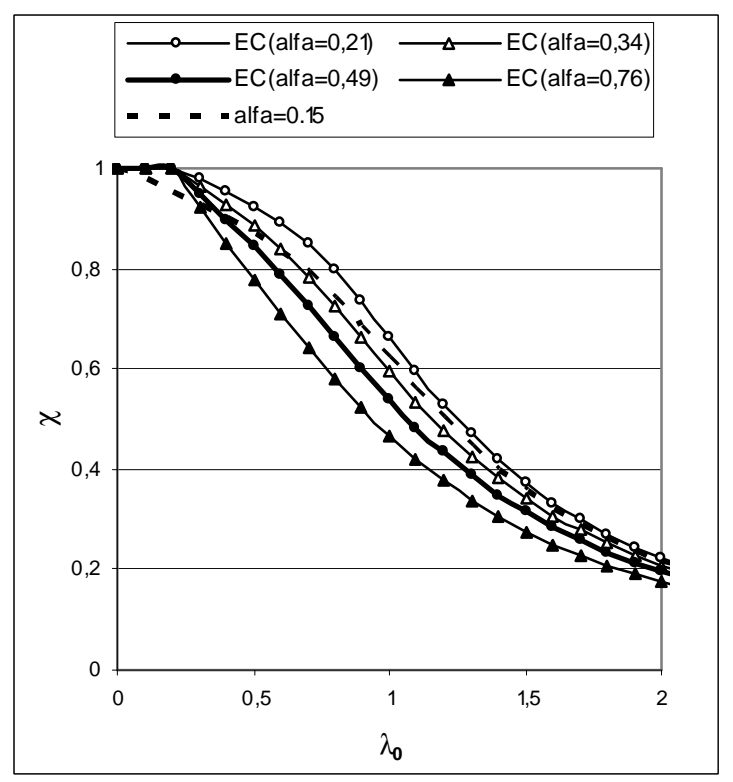

Figura 21: Relação entre $\chi$ e $\lambda_{0}$ conforme Eurocode 3 e para material elástico com imperfeição $\quad(\alpha=0,15)$ geométrica

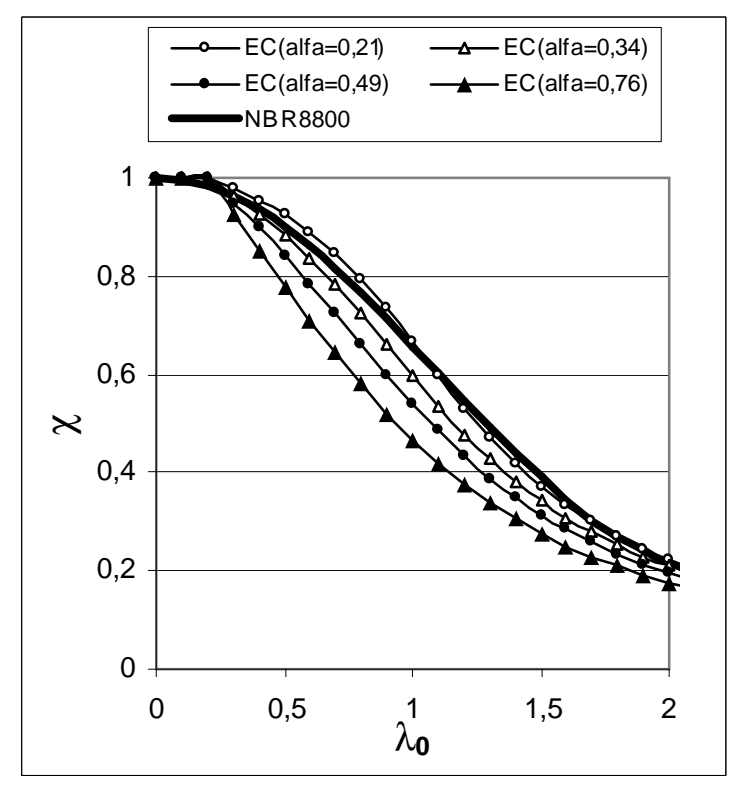

Figura 22: Relação entre $\chi$ e $\lambda_{0}$ conforme NBR 8800 e Eurocode 3

Na figura 24, compara-se a NBR 8800:2008 aos resultados do estudo anterior (item 2.2) válido para material elástico-linear. Apesar de as hipóteses para a dedução da curva da NBR 
8800:2008, que tem por base o AISC (2005), incluírem imperfeições (Pimenta 1997), ela se ajusta perfeitamente à média dos valores determinados para material elástico-linear.

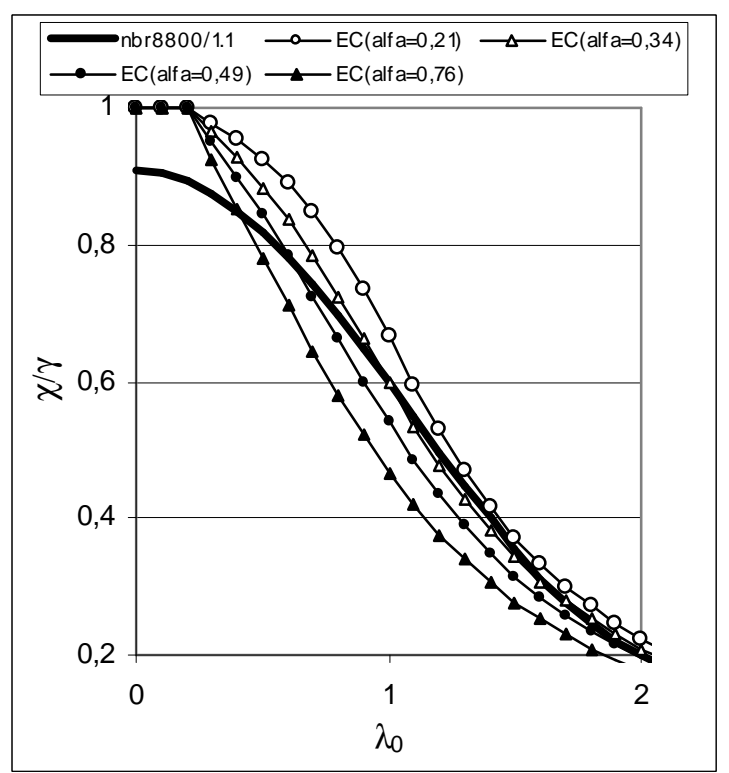

Figura 23: Relação entre $\chi$ e $\lambda_{0}$ conforme NBR 8800 e Eurocode 3

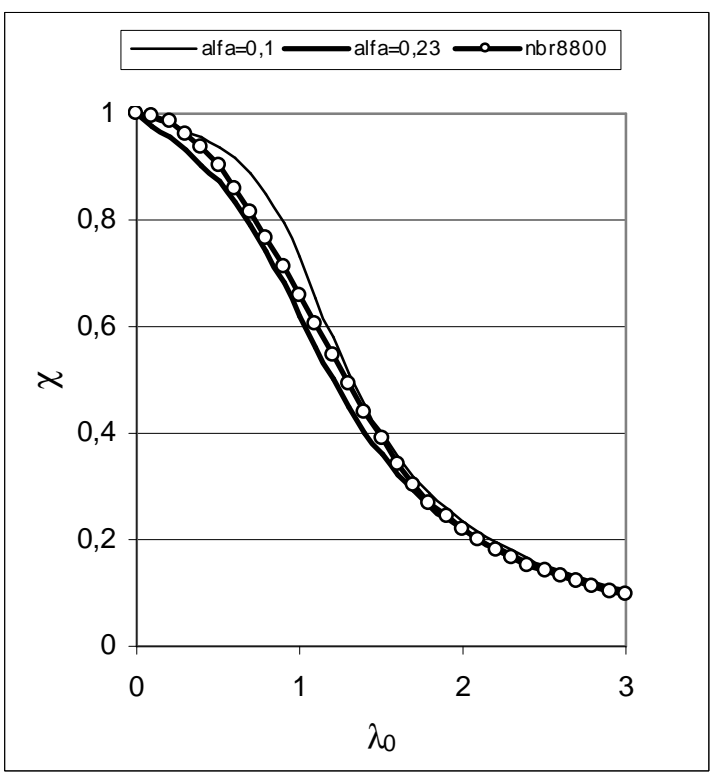

Figura 24: Relação entre $\chi$ e $\lambda 0$ conforme NBR 8800 e para material elástico linear

\section{PILARES EM SITUAÇÃO DE INCÊNDIO}

\subsection{Curva de dimensionamento}

A altas temperaturas, ocorre redução da resistência e do módulo de elasticidade dos materiais. Os valores dos respectivos redutores, $\mathrm{k}_{\mathrm{y}, \theta}$ e $\mathrm{k}_{\mathrm{E}, \theta}$, são indicados na ABNT NBR 14323:1999. O valor de cálculo da força normal resistente em situação de incêndio é determinado por meio da eq. 22 , em que $\gamma_{\mathrm{fi}}$ é o coeficiente de ponderação da resistência em situação de incêndio e igual a 1.

$$
\mathrm{N}_{\mathrm{Rd}, \mathrm{fi}}=\chi_{\mathrm{fi}} \frac{\mathrm{Ak}_{\mathrm{y} \theta} \mathrm{f}_{\mathrm{y}}}{\gamma_{\mathrm{fi}}}
$$

Através dos últimos 25 anos, foram desenvolvidos vários estudos para a determinação de

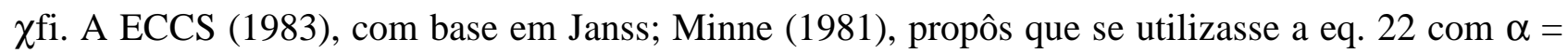
0,49 para qualquer seção de perfil. Nessa publicação era reconhecido que $\lambda 0$ deveria ser substituído por $\lambda 0$,fi (eq. 23), no entanto, à época admitiu-se que $\lambda 0$,fi $=\lambda 0$. A ECCS (1985) manteve a recomendação anterior. O Eurocode 3 (1993) manteve a eq. 20, no entanto, incluiu um fator empírico de correção para reduzir a força normal resistente, tudo se passava como se $\chi_{\mathrm{fi}}=\chi / 1,2$. Manteve ainda $\lambda_{0, \mathrm{fi}}=\lambda_{0}$. O Eurocode 3 (1995) manteve a eq. 20 e o fator 1,2, mas no lugar de $\lambda_{0}$ 
utiliza $\lambda_{0, \text { fi }}$ (eq. 23). Esse procedimento é o recomendado pela ABNT NBR 14323:1999. Franssen, Schleich, Cajot (1995) propuseram uma curva própria para incêndio (eq. 24) alterando a forma de determinar o valor de $\alpha$ (eq. 25). $\beta=1,2$ foi escolhido com base em análise numérica via programa de computador SAFIR, de forma a estar a favor da segurança para todas as análises. Franssen et al. (1996) com base em cerca de 80 resultados experimentais, mantiveram a curva anterior, no entanto alteraram o fator $\beta$ de 1,2 para 0,65 , por ser este o valor médio encontrado. $\mathrm{O}$ código modelo do ECCS (2001) incorpora a proposta de Franssen et al. (1996), que, finalmente também é incluído no Eurocode 3 (2001) e mantém-se até a última versão (Eurocode 3 2005).

$$
\begin{gathered}
\lambda_{0, \mathrm{fi}}=\frac{\lambda}{\sqrt{\pi^{2} \frac{\mathrm{k}_{\mathrm{E}, \theta} \mathrm{E}}{\mathrm{k}_{\mathrm{y}, \theta} \mathrm{f}_{\mathrm{y}, \theta}}}} \\
\chi=\frac{1}{0,5\left[1+\alpha \lambda_{0, \mathrm{fi}}+\lambda_{0, \mathrm{fi}}^{2}\right]+\sqrt{\left[0,5\left(1+\alpha \lambda_{0, \mathrm{fi}}+\lambda_{0, \mathrm{fi}}^{2}\right)\right]^{2}-\lambda_{0, \mathrm{fi}}^{2}}} \\
\alpha=\beta \sqrt{\frac{235}{\mathrm{f}_{\mathrm{y}}}}, \mathrm{f}_{\mathrm{y}} \text { em MPa }
\end{gathered}
$$

Convém esclarecer que o redutor de resistência, $\mathrm{k}_{\mathrm{y}, \theta}$, apresentado na ABNT NBR 14323:1999 corresponde à redução da resistência ao escoamento $\mathrm{f}_{\mathrm{y}}$. No caso de pilares, o estado limite último por instabilidade global, geralmente, ocorre antes de o escoamento ser atingido. Ao invés de alterar o valor de $\mathrm{k}_{\mathrm{y}, \theta}$, as normas alteraram a forma de determinação da $\mathrm{N}_{\mathrm{Rd}, \mathrm{fi}}$. O Eurocode adotou uma nova curva $\chi_{\mathrm{fi}} \times \lambda_{0, \mathrm{fi}}$ (eqs. 23 a 25) e a NBR 14323:1999 adotou o fator de correção 1,2. Da mesma forma, $\mathrm{o} \mathrm{k}_{\mathrm{y}, \theta}$ conforme a norma brasileira não considera a possibilidade de instabilidade local. Para os casos em que esse fenômeno seja o preponderante, valores menores de $\mathrm{k}_{\mathrm{y}, \theta}$ devem ser empregados (Eurocode 3 2005, Renaud; Zhao 2006). Para fins de comparação, plotam-se nas figuras 25 e 26 os valores de $\chi_{\text {fi }}$ em função de $\lambda_{0}$ conforme as várias hipóteses aqui citadas. Para a construção desses gráficos adotou-se, por simplicidade, $\lambda_{0, \mathrm{fi}}=\lambda_{0} / 0,85$.

As normas europeias são conhecidas pela racionalidade das expressões normatizadas. Seguindo essa linha, as expressões para dimensionamento recomendadas pelo Eurocode 3 (2003) "à temperatura ambiente" e EUROCODE 3 (2005) "em incêndio" são formalmente parecidas. Essa similaridade ocorria também entre as normas ABNT NBR 14323:1999 e ABNT NBR 8800:1986. No entanto, a ABNT NBR 8800:2008 alterou a forma de dimensionar pilares à temperatura ambiente, seguindo o AISC (2005) (eq. 21 deste texto). Isso tornou a expressão muito diferente daquela da ABNT NBR 14323:1999. Os autores deste texto tentaram obter uma expressão para dimensionamento de pilares em incêndio, com alguma similaridade àquela recomendada pela ABNT NBR 8800:2008, a fim de sugerir sua inclusão na revisão da NBR 14323. Na figura 27, 
observa-se a relação entre os resultados obtidos para $\chi_{\text {fi }}$, determinado por meio da eq. 24 (Eurocode 3), para $\mathrm{f}_{\mathrm{y}}=250 \mathrm{MPa}$ e de $\chi$ determinado pela eq. 21 (ABNT NBR 8800:2008) adotando-se $\lambda_{0, \mathrm{fi}}$ no lugar de $\lambda_{0}$. Apesar da grande variabilidade, adotou-se, a favor da segurança, um fator de proporcionalidade de 1,4 .

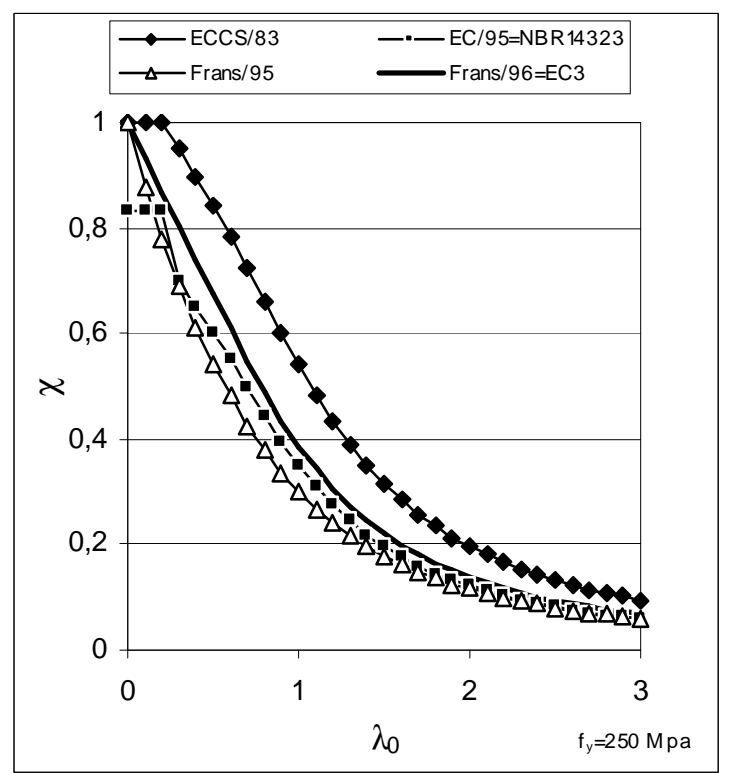

Figura 25: Curvas de dimensionamento de pilares em incêndio para $\mathrm{f}_{\mathrm{y}}=250 \mathrm{MPa}$

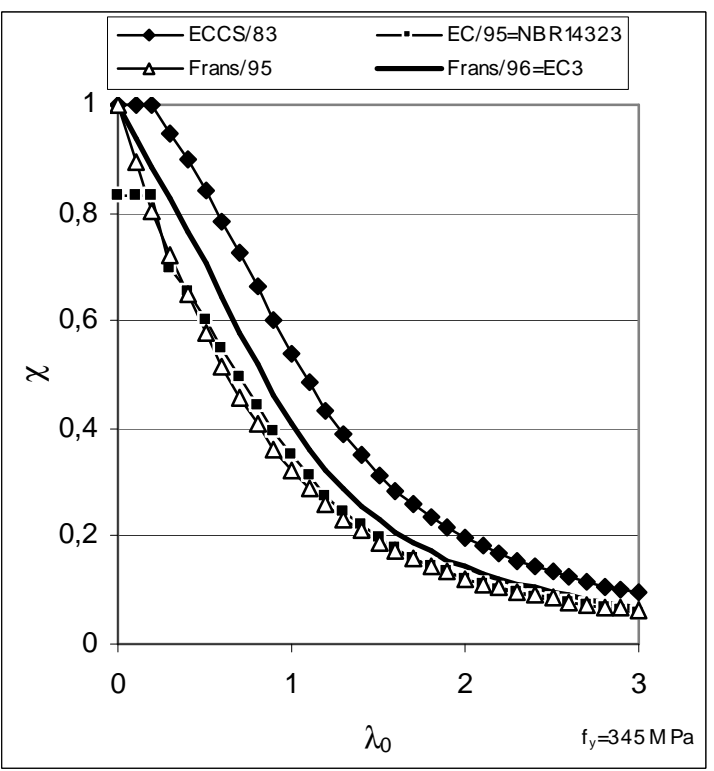

Figura 26: Curvas de dimensionamento de pilares em incêndio para $\mathrm{f}_{\mathrm{y}}=345 \mathrm{MPa}$

Determinando-se $\chi_{\text {fi }}$ por meio da eq. 21 (NBR 8800:2008) usando-se $\lambda_{0, \text { fi }}$ no lugar de $\lambda_{0}$, e dividindo-se o resultado final por 1,4 , encontra-se a curva $\chi_{\mathrm{fi}} \mathrm{x} \lambda_{0}$, apresentada na figura 28 juntamente com a determinada diretamente pela expressão do Eurocode 3 (eq. 26). Ajustando-se a curva, com base na ABNT NBR 8800:2008, para valores inferiores a $\lambda_{0, \mathrm{fi}}=0,8$ encontra-se a eq. 26. $\mathrm{Na}$ figura 29, comparam-se os resultados da eq. 26 aos da eq. 24. Na figura 30 vêm-se os erros cometidos com essa aproximação.

$$
\begin{aligned}
& \operatorname{para} \lambda_{0, \mathrm{fi}}=0 \rightarrow \chi_{\mathrm{fi}}=1,0 \\
& \text { para } 0<\lambda_{0, \mathrm{fi}} \leq 0,8 \rightarrow \chi=1-0,567 \lambda_{0, \mathrm{fi}} \\
& \text { para } 0,8<\lambda_{0, \mathrm{fi}} \leq 1,5 \rightarrow \chi=0,658^{\lambda_{0, \mathrm{fi}}^{2}} \\
& \text { para } \lambda_{0, \mathrm{fi}}>1,5 \rightarrow \chi=\frac{0,877}{\lambda_{0, \mathrm{fi}}^{2}}
\end{aligned}
$$

Apesar de a eq. 26 concordar com a curva do Eurocode 3 (eq. 24), transparecem os ajustes feitos com muitos intervalos e, ainda assim, com formulação diferente. Os autores recomendam que, para a revisão da ABNT NBR 14323:1999, seja mantida a base original, qual seja, o Eurocode 3 (2005) que, entre outras vantagens, apresenta uma curva deduzida especificamente para a situação de incêndio. 


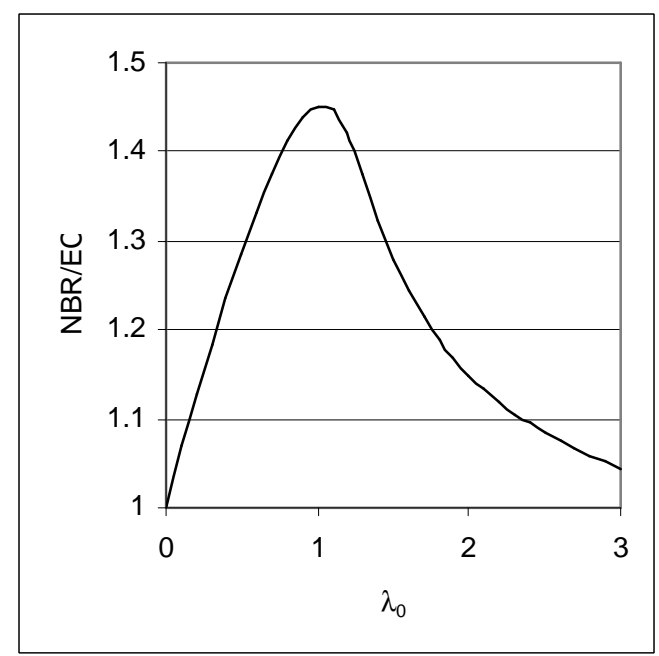

Figura 27: Relação $\chi_{\mathrm{fi}}$ (Eurocode) e $\chi$ (NBR 8800)

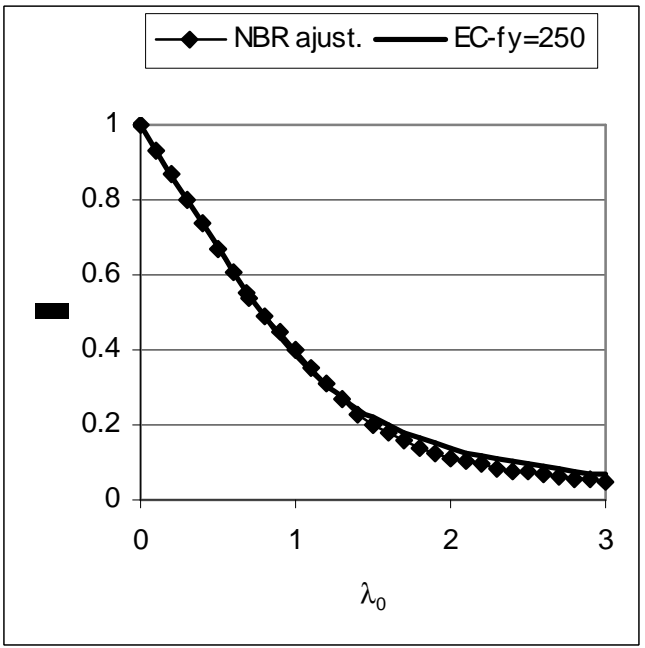

Figura 29: Curva $\chi_{\mathrm{fi}} \mathrm{x} \lambda_{0}$ determinada pelo Eurocode e pela NBR 8800 ajustada

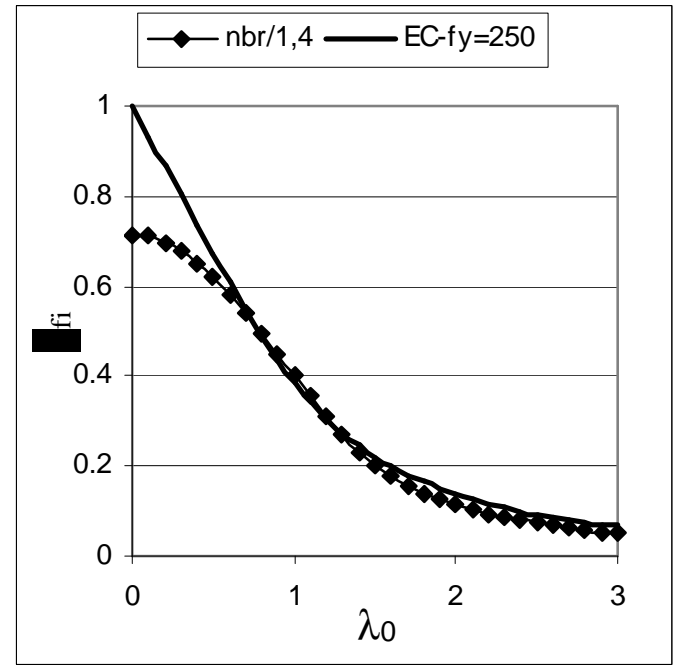

Figura 28: Gráficos $\chi_{\mathrm{fi}} \times \lambda_{0}$ com base na NBR8800 dividindo-se os resultados por 1,4 e no Eurocode

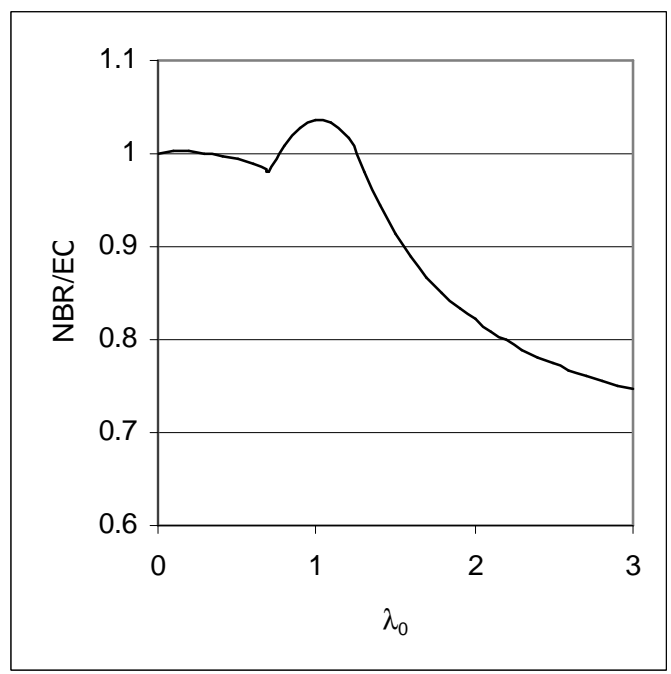

Figura 30: Relação entre $\chi_{\mathrm{fi}}$ determinado pelo Eurocode e pela NBR 8800 ajustada

\subsection{Temperatura crítica de pilares}

A temperatura crítica $\theta_{\text {cr }}$ de um elemento estrutural é a sua temperatura de colapso. Verificase a segurança de um elemento estrutural em situação de incêndio por meio da eq. 27 , onde $\theta_{a}$ é a temperatura do aço.

$$
\theta_{a} \leq \theta_{c r}
$$

A eq. 27, obviamente, somente é aplicada se a distribuição de temperaturas for uniforme em todo o volume do elemento estrutural, por exemplo, em pilares de aço isolados. $\mathrm{O}$ valor de $\theta_{\text {a }}$ pode ser calculada por meio de ensaios ou analiticamente (Silva 2005a, 2005b). O valor de $\theta_{\text {cr }}$ pode ser calculado por meio de modelagens numéricas ou por métodos simplificados de dimensionamento (NBR 14323:1999; Silva e Fakury 2001; Silva 2004). A partir das eqs. 28 e 29, em que $\mathrm{N}_{\mathrm{Rd}}$ e $\mathrm{N}_{\mathrm{Rd}, \mathrm{fi}}$ 
são, respectivamente, o valor de cálculo da força normal resistente à temperatura ambiente e em situação de incêndio e $\gamma$ e $\gamma_{\mathrm{fi}}$ são, respectivamente, o coeficiente de ponderação da resistência à temperatura ambiente e em incêndio, tem-se que $\mathrm{N}_{\mathrm{Rd}, \mathrm{fi}}=\chi_{\mathrm{fi}} \mathrm{N}_{\mathrm{Rd}} \gamma \mathrm{k}_{\mathrm{y} \theta} /\left(\chi \gamma_{\mathrm{fi}}\right)$. Rearranjando, obtémse $\mathrm{N}_{\mathrm{Rd}, \mathrm{fi}} / \mathrm{N}_{\mathrm{Rd}}=\mathrm{k}_{\mathrm{y} \theta}\left(\chi_{\mathrm{fi}} \gamma\right) /\left(\chi \gamma_{\mathrm{fi}}\right)$. Lembrando-se que $\gamma / \gamma_{\mathrm{fi}}=1,1$ e que na temperatura crítica $N_{R d, f i}=N_{S d, f i}$, encontra-se a eq. 30 , em que $\eta=N_{f i, S d} / N_{R d}$, sendo $N_{f i, S d}$ o valor de cálculo da força normal atuante, em situação de incêndio.

$$
\begin{gathered}
\mathrm{N}_{\mathrm{Rd}}=\chi \frac{\mathrm{Af}_{\mathrm{y}}}{\gamma} \\
\mathrm{N}_{\mathrm{Rd}, \mathrm{fi}}=\chi_{\mathrm{fi}} \frac{\mathrm{Ak}_{\mathrm{y} \theta} \mathrm{f}_{\mathrm{y}}}{\gamma_{\mathrm{fi}}}
\end{gathered}
$$

$\mathrm{N}_{\mathrm{fi}, \mathrm{Sd}}$ é facilmente calculado a partir das combinações de ações fornecidas pela ABNT NBR 8681:2003 ou ABNT NBR 14323:1999. $\mathrm{N}_{\mathrm{Rd}}$ e $\lambda_{0}$ são valores conhecidos, pois são utilizados no dimensionamento à temperatura ambiente.

$$
\eta=\frac{\chi_{\mathrm{fi}}}{\chi} 1,1 \mathrm{k}_{\mathrm{y} \theta}
$$

Considerando-se que $\chi$ é função de $\lambda_{0}, \chi_{\mathrm{fi}}$ é função de $\lambda_{0, \mathrm{fi}}$ que por sua vez é função de $\lambda_{0} \mathrm{e} \theta$ e $k_{y, \theta}$ é função de $\theta$, da eq. 30 tem-se que $\theta_{\mathrm{cr}}=\mathrm{f}\left(\chi, \lambda_{0}\right)$. Em vista de essa função ser complexa, desenvolveu-se um programa de computador para resolver a eq. 30 aplicada a vários casos de $\lambda_{0} \mathrm{e}$ $\eta$. Em Silva (2004) foram apresentadas curvas que associavam a temperatura crítica de pilares aos valores do índice de esbeltez reduzido e do nível de carregamento do pilar. Essas curvas foram recalculadas em Velarde (2008) considerando-se a ABNT NBR 8800:2008 para o dimensionamento de pilares à temperatura ambiente e o Eurocode 3 (eq. 24) para o dimensionamento de pilares em situação de incêndio. Tais gráficos são apresentados às figuras 31 a 33. 


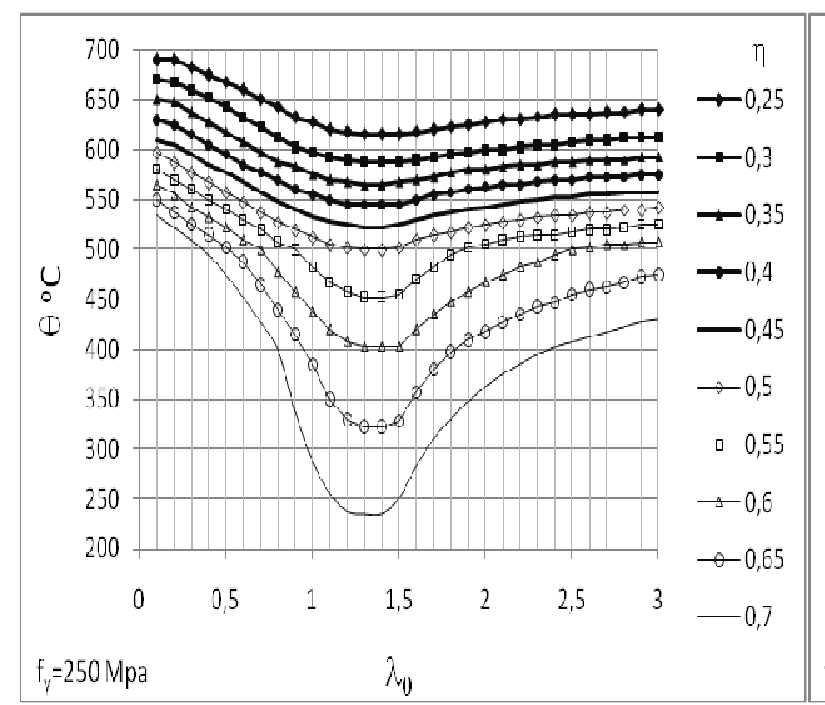

Figura 31: Temperatura crítica de pilares de aço $\operatorname{com} \mathrm{f}_{\mathrm{y}}=$ $250 \mathrm{MPa}$

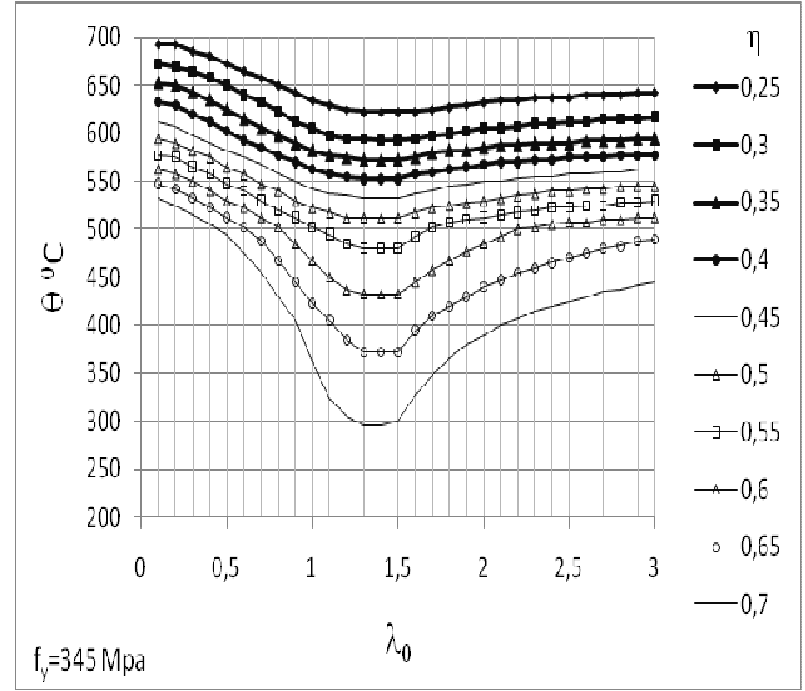

Figura 33: Temperatura crítica de pilares de aço com $\mathrm{f}_{\mathrm{y}}=$ $345 \mathrm{MPa}$

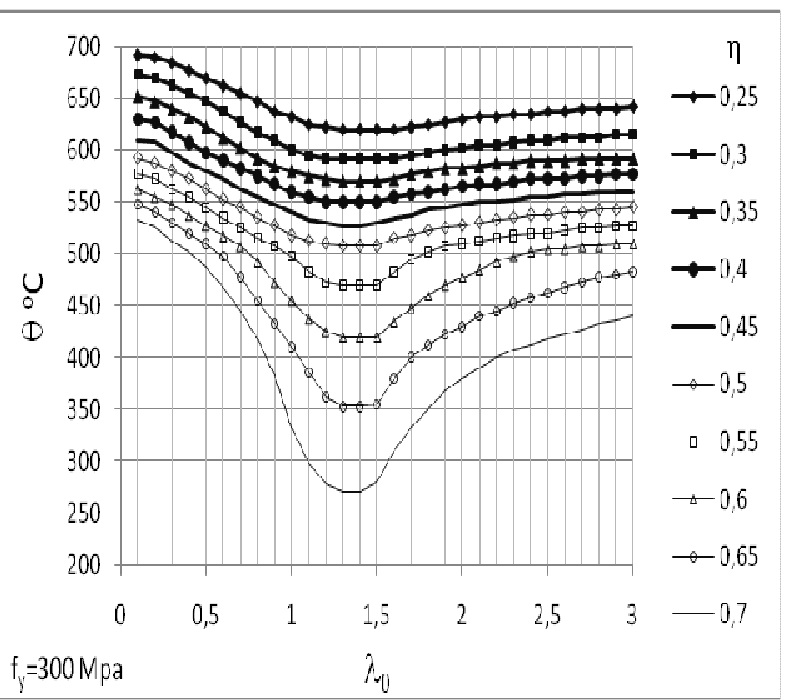

Figura 32: Temperatura crítica de pilares de aço $\operatorname{com} \mathrm{f}_{\mathrm{y}}=$ $300 \mathrm{MPa}$

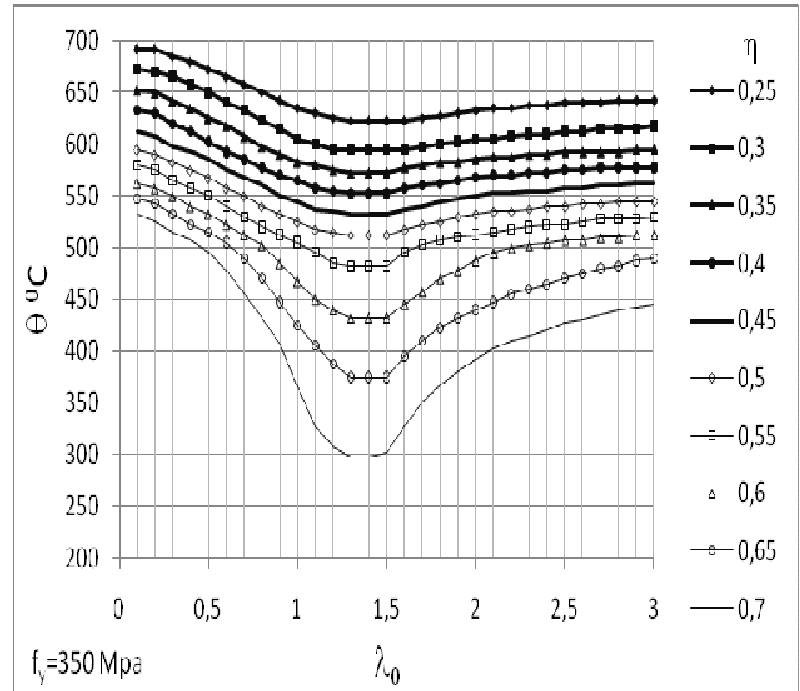

Figura 32: Temperatura crítica de pilares de aço $\operatorname{com~} \mathrm{f}_{\mathrm{y}}=$ $350 \mathrm{MPa}$

Nota-se um ponto de mínimo para valores de esbeltez entre 1 e 1,5 , podendo dar a falsa impressão de que a temperatura crítica possa aumentar com o índice de esbeltez. Ocorre que cada curva está associada a um determinado nível de carregamento $\eta$, que também se altera se $\lambda_{0}$ variar.

\section{CONCLUSÃO}

Analisou-se neste trabalho a variação da força normal resistente de pilares de aço em função da esbeltez, para vários modelos constitutivos, incluindo imperfeições geométricas. Mostrou-se, via análise de estabilidade do equilíbrio de pilares com rigidez concentrada, que o estado limite último é ou ponto limite sem reversão ou escoamento, não ocorrendo flambagem (bifurcação) em pilares reais. O redutor da força resistente de um pilar com comportamento elástico, com imperfeição 
geométrica e sob força centrada foi deduzido. Mostrou-se como esse redutor-padrão originou a curva europeia de dimensionamento em incêndio. Finalmente recomendou-se empregar o Eurocode 3 na revisão da ABNT NBR 14432:1999.

Foram construídos gráficos para a determinação expedita da temperatura crítica de pilares, com base no Eurocode 3, em função da esbeltez reduzida em temperatura ambiente e do nível de carregamento.

\section{REFERÊNCIAS BIBLIOGRÁFICAS}

AMERICAN INSTITUTE OF STEEL CONSTRUCTION (AISC). Specification for structural steel buildings. 2005. Chicago.

ASSOCIAÇÃO BRASILEIRA DE NORMAS TÉCNICAS. Ações e segurança nas estruturas. ABNT NBR 8681: 2003. Rio de Janeiro.

Projeto de estruturas de aço e de estruturas mistas de aço e concreto de edifícios. ABNT NBR 8800:2008. Rio de Janeiro.

Projeto de estruturas de aço de edifícios. ABNT NBR 8800:1986. Rio de Janeiro.

. Dimensionamento de estruturas de aço de edifícios em situação de incêndio. ABNT

NBR 14323:1999. Rio de Janeiro.

EUROPEAN COMMITTEE FOR STANDARDIZATION. Eurocode 3. Design of steel structures part 1.2 structural fire design. prENV 1993-1-2. 1993, Brussels.

1995, Brussels.

Eurocode 3. Design of steel structures part 1.2 structural fire design. ENV 1993-1-2.

Eurocode 3. Design of steel structures part 1.2 General rules - Structural fire design. Draft prEN 1993-1-2. 2001. Brussels.

Eurocode 3. Design of steel structures. Part 1.1 General Rules and rules for building. prEN 1993-1-1. 2003. Brussels.

. Eurocode 3. Design of steel structures part 1.2 General rules - Structural fire design. BS EN 1993-1-2. 2005.

EUROPEAN CONVENTION FOR CONSTRUCTIONAL STEELWORK - ECCS. Technical Committee 3, Fire Safety of Steel Structures. European recommendation for the fire safety of steel structures. Elsevier. Scientific Publishing Company. 1983. Amsterdam.

. Technical Committee 3, Fire Safety of Steel Structures. Design manual on the European recommendation for the fire safety of steel structures. 1985. Brussels.

. Technical Committee 3. Model code on fire engineering. 2001. Brussels. 
FRANSSEN, J. M.; SCHLEICH, J. B.; CAJOT, L. G. A simple model for the fire resistance of axially loaded members according to Eurocode 3. Journal of Constructional Steel Research, v. 35. 1995. Malta.

FRANSSEN, J. M.; SCHLEICH, J. B.; CAJOT, L. G.; AZPIAZU, W. A Simple Model for the Fire Resistance of Axially Loaded Members - Comparison with Experimental Results. Journal of Constructional Steel Research. v. 37. 1996. Great Britain.

FRUCHTENGARTEN, J. Notas de aula. Escola Politécnica da USP. 1988. São Paulo.

JANSS, J.; MINNE, R. Buckling of steel columns in fire conditions. Fire Safety Journal, v. 4. 1981. Amsterdam.

PIMENTA, R. J. Proposição de uma curva de flambagem para perfis I soldados formados por chapas cortadas a maçarico. Dissertação de mestrado apresentado à Escola de Engenharia da Universidade Federal de Minas Gerais. 1997. Belo Horizonte.

REIS. A.; Camotim, D. Estabilidade estrutural. McGraw Hill. 2001. Lisboa.

RENAUD, C.; ZHAO, B. Investigation of simple calculation method in EN 1993-1-2 for buckling of hot rolled class 4 steel members exposed to fire. In: Fourth Workshop Structures in Fire. 2006. Aveiro.

RONDAL, J.; MAQUOI, R. Single equation for SSRC columns strength curves. Journal of the structural division (ASCE). v. 105. n. St 1. 1979.

SILVA, V. P. Estruturas de aço em situação de incêndio. Zigurate Editora. 2004. São Paulo.

. Determination of the steel fire protection material thickness by analytical process A simple derivation. Engineering Structures. v. 27. 2005a.

Determination of the temperature of thermally unprotected steel members under

fire situations: considerations on the section factor. Latin American Journal of Solids and Structures v. 3. 2005b.

SILVA, V. P.; FAKURY, R. H. Brazilian Standards for steel structures fire design. Fire Safety Journal, v. 37. 2001.

VELARDE, J. S. S. Sobre o comportamento de pilares de aço em situação de incêndio. Dissertação de Mestrado apresentada à Escola Politécnica da Universidade de São Paulo. 2008. São Paulo.

\section{AGRADECIMENTOS}

Os autores agradecem o apoio recebido do CBCA- Centro Brasileiro da Construção em Aço, do CNPq - Conselho Nacional de Desenvolvimento Científico e Tecnológico e da FAPESP Fundação para Amparo à Pesquisa do Estado de São Paulo. 


\title{
DESIGN OF COLUMNS AT ROOM TEMPERATURE AND IN FIRE SITUATION
}

\begin{abstract}
EXTEND
The design of steel columns at room temperature recommended by Brazilian standard ABNT NBR 8800:1986 was based on the European multiple curves. These curves relate the column non-dimensional slenderness $\left(\lambda_{0}\right)$ to a reduction factor of the column resistance. The Brazilian standard ABNT NBR 14323:1999 "Steel structures fire design" also follows the European standard for columns fire design. There is, therefore, similarity between the formulations of both standards. The review of NBR 8800, i. e., ABNT NBR 8800:2008, however, was based on the North American specification AISC (American Iron and Steel Construction). The North American and European room temperature curves although lead to similar results, their formulations are totally different. The objective of this work is to propose that the revision of NBR 14323 keeps the European base for the columns fire design. To lead to this proposal, one is presented detailed and didactic introduction on the behavior of columns at room temperature. In Chapter 1 are included topics related to the classic buckling theory. In chapter 2 the effects of the geometric imperfections and of the material behavior applied to elasto-fragile material are considered and the equation of Perry-Robertson (eq. 15) is derived. The same study applied to elastoplastic perfect and non-linear materials is done in chapter 3. For all studies, resistance reduction factor vs non-dimensinal slendereness curves are shown. In chapter 4, a simplified but interesting study of the stability of the columns with concentrated rigidity based on the minimization of the total potential energy is presented. The origin of the European curves for room temperature and comparisons between results from Eurocode, AISC (equal ABNT NBR 8800:2008) and equation 15 are presented in chapter 5. The origin of the European curve for fire design is presented in chapter 6. Attempts to adjust the North American curve to the European have been made without success, as expected. The authors recommend that, for the revision of ABNT NBR 14323:1999, be kept the original base, i. e., Eurocode that, among others advantages, presents a curve specifically deduced for the fire situation. Graphics for the easy determination of columns critical temperature are included in figures 31 to 34 .
\end{abstract}

Key words: Fire. Columns. Instability.

\section{CONCLUSION}

The variation of the compression force resistance of steel columns in function of the slenderness, for some constitutive models, including geometrical imperfection was analyzed in this work. The ultimate limit state of a real column is the yield or the limit point buckling and not the bifurcation buckling was showed. It was analyzed the evolution of the expressions of the curves of reduction of compression force resistance of columns in fire. It was recommended the use of the Eurocode curve for revision of the Brazilian standard NBR 14323 - Steel structures fire design. It was produced graphs for the fast determination of the columns critical temperature, based on Eurocode 3, in function of the reduced slenderness and load level. 